\title{
Aguacate Hass, la ruta de las pérdidas económicas
}

\section{Hass avocado, the route of economic losses}

DOI: $10.46932 / \operatorname{sfjdv3n1-024}$

Received in: Dec 30st, 2021

Accepted in: Jan 1th, 2022

\section{Claudia Verónica Arreola Macías}

Estudiante de ingeniería gestión empresarial. instituto tecnológico de Álvaro Obregón

E-mail: 1181210114@aobregon.tecnm.mx

\section{Miguel Ángel Camacho Rosales}

Estudiante de ingeniería gestión empresarial. instituto tecnológico de Álvaro Obregón

E-mail: 1181210123@aobregon.tecnm.mx

\section{Liliana Ocampo Bautista}

Estudiante de ingeniería gestión empresarial. instituto tecnológico de Álvaro Obregón

E-mail: 1181210116@aobregon.tecnm.mx

\section{Diana Berenice Rico Díaz}

Estudiante de ingeniería gestión empresarial. instituto tecnológico de Álvaro Obregón

E-mail:1181210088@aobregon.tecnm.mx

Hernández Noé Rocha

Estudiante de ingeniería gestión empresarial. instituto tecnológico de Álvaro Obregón

E-mail: 1181210084@aobregon.tecnm.mx

\section{María Fernanda Santos Hernández}

Estudiante de ingeniería gestión empresarial. instituto tecnológico de Álvaro Obregón

E-mail: 1181210183@aobregon.tecnm.mx

\section{Juan Carlos Sartorius Contreras}

Estudiante de ingeniería gestión empresarial. instituto tecnológico de Álvaro Obregón

E-mail: 1181210192@aobregon.tecnm.mx

\section{RESUMEN}

La industria del aguacate está creciendo cada vez más, existiendo nula protección para los campesinos ante la delincuencia organizada. En las fuentes obtenidas se recuperó la entrevista de un hombre cuya familia tiene dos granjas de aguacate, pero incluso él tuvo miedo de que su nombre se publicara, por miedo. En el municipio de Uruapan Michoacán, se concentra $84.9 \%$ del volumen de producción de 
aguacate en México, ya que produce más de un millón de toneladas de aguacate para tu comercialización. Los agricultores producen tres tipos de aguacate en la región son: Hass, Criollo y Fuerte (APEAM, 2019). El método Winter nos permite realizar un pronóstico a través de un análisis de serie de tiempo, para nuestro caso nos permite analizar el comportamiento de las pérdidas económicas mensuales por consecuencia del crimen organizado, destacando así, que trimestre se encuentra en mayor riesgo de pérdidas y, reduciendo drásticamente el resultado final del año 2021.

Palabras clave: Pérdidas económicas, Auge, Crimen organizado, Comercialización.

\begin{abstract}
The avocado industry is growing and there is no protection for farmers from organized crime. organized crime. Sources recovered an interview with a man whose family has two avocado farms, but even he was afraid to have his name published, out of fear. In the municipality of Uruapan Michoacán, $84.9 \%$ of the avocado production volume is concentrated in the municipality of Uruapan Michoacán. of the volume of avocado production in Mexico, producing more than one million tons of avocados for commercialization. marketing. Farmers produce three types of avocado in the region: Hass, Criollo and Fuerte (APEAM, 2019). The Winter method allows us to perform a forecast through a time series analysis, for our case it allows us to the behavior of monthly economic losses as a result of organized crime, highlighting which quarter is at the highest risk of economic losses due to organized crime. quarter is at greater risk of losses and, drastically reducing the final result of the year 2021 .
\end{abstract}

Keywords: Economic losses, Boom, Organized crime, Commercialization.

\title{
1 INTRODUCCIÓN
}

Donde hay dinero, está el crimen. Por eso no resulta extraño escuchar que el crimen organizado está tratando de apoderarse de los campos de aguacate en México, específicamente los cultivos del estado de Michoacán Uruapan, después de que la popularidad del aguacate creciera alrededor del mundo, y que su precio se disparara por una mayor demanda.

En la presente investigación, se analizará que cada vez es más común que grupos de crimen organizado se alejen de drogas y se enfoquen en los campos de aguacates, enfocándose en las rutas que toman los cultivadores y proveedores, para asaltarlos, y redirigir el producto hacia sus propias rutas, las cuales van en su mayoría hacia Estados Unidos, donde cada vez existe más demanda. Esto porque la industria del aguacate está creciendo cada vez más, y no existe protección para los campesinos. En las fuentes obtenidas se entrevistó a un hombre cuya familia tiene dos granjas de aguacate, pero incluso él tuvo miedo de que su nombre se publicara, por miedo. El hombre declaró,

“Tratamos de trabajar con el gobierno, pero ni ellos se atreven a entrar a ciertas áreas" (Alonzo M. febrero 7 de 2020. El oro verde).

Lo que más resaltan los habitantes de estas comunidades es que casi no existe policía que proteja a los productores. En Uruapan cuentan con poca seguridad, y no protegen el cultivo para ser comercializado. Si no se comienza a abordar este tema, podríamos ver cómo el crimen organizado se 
apodera de una parte importante de la producción de alimentos en México, quitándole trabajos a decenas familias que lo necesitan.

Entre 2001 y 2010, la producción de aguacate en el estado de Michoacán especialmente en el municipio de Uruapan, que abarca más del 70\% de las cosechas del país, se triplicó, y las exportaciones se multiplicaron por diez, según un informe publicado en 2012 por el instituto Tapia Vargas. El informe sugiere que la expansión causó la pérdida de tierras de aproximadamente 690 hectáreas al año entre 2000 y 2010 .

\subsection{PLANTEAMIENTO DEL PROBLEMA.}

En el municipio de Uruapan Michoacán, se concentra $84.9 \%$ del volumen de producción de aguacate en México, ya que produce más de un millón de toneladas de aguacate para tu comercialización. Los agricultores producen tres tipos de aguacate en la región son: Hass, Criollo y Fuerte (APEAM, 2019).

El aguacate es uno de los productos más exitosos de la exportación agroalimentaria nacional. México es el principal exportador del mercado internacional con una aportación de $45.95 \%$ del valor de las exportaciones.

El municipio de Uruapan Michoacán, su principal fruto con más éxito en la región, es el aguacate hass y ha presentado un gran auge para su comercialización y exportación por la calidad del fruto. Pero ha despertado el interés por el aguacate hass a organizaciones delictivas por su gran éxito en el mercado y su alza de ventas año con año y su gran margen de ganancia para los agricultores de la región.

La principal problemática de los agricultores de aguacate hass es el robo del fruto por parte del crimen organizado, el robo de aguacate representa pérdidas económicas de más de 2 millones de pesos para los agricultores de Michoacán, por que registran de 7 a 10 camiones hurtados a la semana por parte del crimen organizado. Cada camión carga 8 toneladas de aguacate hass, cuyo costo promedio es de 200 mil pesos. (Excelsior 2019).

La afectación es muy grande porque a lo mejor ya llevamos varios años con este problema, pero no decrece, hay momentos en los que si se ha tomado una cierta calma, pero hasta el momento sigue siendo muy grande la afectación porque nos están robando entre 7 o 10 camiones por semana”, explicó David Ruiz, vocal del sector empacador APEAM. (2019).

Las afectaciones han sido severas para los agricultores de Uruapan Michoacán, por el robo de sus camiones llenos de aguacate, la afectación es de tipo económico ya que previamente se estimará las pérdidas económicas tomando como base el año 2019 y así proyectar las pérdidas por el robo de camiones del fruto para el año 2021. 


\subsubsection{Justificación}

Dar a conocer las afectaciones económicas que han pasado los agricultores de aguacate hass, tras el auge que se ha presentado en la comercialización del fruto, en el estado de Michoacán principalmente en el municipio de Uruapan por los robos de los camiones llenos de aguacate hass estos robos son incontrolables del crimen organizado. El robo de aguacate ha aumentado año con año, en la investigación se estimará las pérdidas económicas para el año 2021, por el robo de camiones llenos de aguacate hass, partiendo como base en el año 2019 se estimará una pérdida económica entre 150 y 200 millones de pesos para el año 2021.

Así, la próxima investigación permitirá demostrar el valor de comercializar aguacate hass, ya que los principales beneficiados de la investigación son otros Estados de México que se dedican a la misma actividad de producir aguacate hass. El estudio próximo servirá para el cuidado del fruto y así prevenir pérdidas económicas.

\subsubsection{Pregunta de investigación}

¿Qué pérdidas económicas generará la delincuencia organizada a los agricultores de aguacate hass del municipio de Uruapan Michoacán para el año 2021?

\subsubsection{Objetivo general}

Proyectar las pérdidas económicas que generará la delincuencia organizada a los agricultores de aguacate hass del municipio de Uruapan Michoacán para el año 2021.

\subsubsection{Objetivos específicos}

- Estimar las pérdidas económicas por robo de camiones de aguacate hass en Uruapan Michoacán en el año 2021, tomando como base el año 2019.

- Graficar las pérdidas económicas por robo de camiones de aguacate hass en el municipio de Uruapan Michoacán en el año 2021, tomando como base el año 2019.

- Realizar los cálculos necesarios para llegar a una proyección de pérdidas económicas para el año 2021, por robo de camiones de aguacates hass, tomando como base cifras del año 2019.

\subsection{HIPÓTESIS}

Tras el auge que se ha presentado en la comercialización del aguacate hass, en el municipio de Uruapan Michoacán, se han presentado robos de camiones de aguacates hass, por lo que se estiman pérdidas económicas entre 150 y 200 millones de peos, en el año 2021. 


\subsubsection{Variable independiente.}

El auge que se ha presentado en la comercialización del aguacate hass.

\subsubsection{Variables dependientes.}

- Robo de camiones.

- Pérdidas económicas.

\section{ESTADO DEL ARTE.}

\subsection{HISTORIA DEL AGUACATE EN MÉXICO.}

La palabra aguacate proviene del náhuatl "ahuacatl", que quiere decir testículo y se refiere a la forma de la fruta. Esto equivale a que se llamase también "fruta de la fertilidad", ya que los aztecas pensaban que tenía poderes especiales, entre ellos cualidades afrodisíacas en hombres y mujeres, además de dar fuerza a quien lo consumiera.

Esta deliciosa fruta fue encontrada en Puebla, en el centro de México, pero los aguacates llevan casi 10,000 años siendo parte de la dieta mesoamericana.

Uruapan es la región líder en la producción de aguacate del estado de Michoacán Se le conoce a esta ciudad como "La Capital Mundial del Aguacate" ya que cuenta con abundante agua y sus tierras son muy fértiles.

\subsection{ANTECEDENTES.}

Uruapan es la región líder en la producción de aguacate del estado de Michoacán, a raíz del auge y el desarrollo de la producción aguacatera Valdez, A. y Sánchez, M. Refiere en su artículo "Oro verde”, que principios 2007 la industria de aguacate en Michoacán se vio afectada por las prácticas de intimidación como asesinatos, secuestros, robo de producto etc. Ejercida; por carteles armados.

Seis años después en el año 2013 la situación se vuelve tan insoportable para los productores aguacateros de Michoacán, armando ellos mismos grupos de autodefensa teniendo como objetivo expulsar a los criminales y recuperar sus centros de producción.

En palabras de José María Ayala Montero, agricultor aguacatero de municipio de Uruapan Michoacán refiere

"La amenaza es constante y de todos lados"

De acuerdo con la FGE la Fiscalía General de Estado de Michoacán Se estima que en el año 2019 se realizaron robos de tan solo 8 toneladas por semana posterior a esto en el año 2020 la OEM INFOMEX reporta el aumento de robo a 10 toneladas por semana. 
Figura 1.

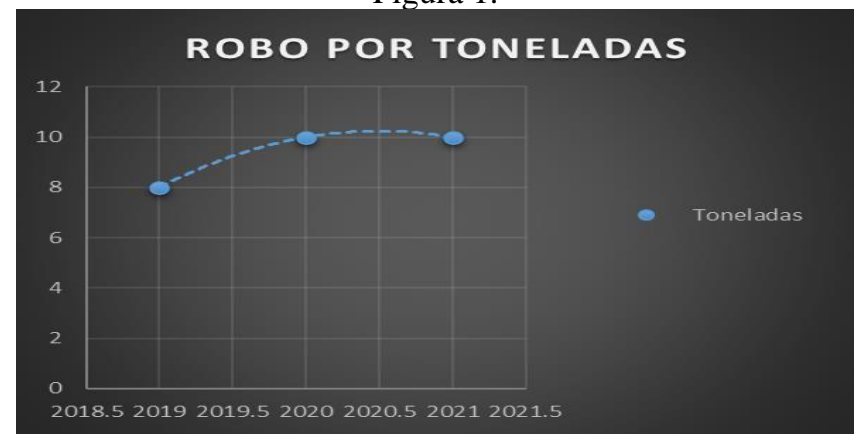

Actualmente se reporta la misma cantidad toneladas perdidas debido de tendríamos casi robo de aguacates por lo que en la gráfica podemos ver como de 2019 a 2020 hay un incremento del robo por toneladas y que para 2021 tenemos la misma cantidad que en el 2020

De la información con la que se cuenta actualmente proporcionada por la FGE las pérdidas económicas ascienden a más de 5 millones de pesos semanalmente ,de acuerdo trabajos de inteligencia e investigación de campo, personal de la Fiscalía General del Estado de Michoacán (FGE) refiere que no todos los robos de camiones de aguacate se reportan a las autoridades esto debido a que algunos agricultores que sufren este tipo de percances se encuentran amenazados o no denuncian por temor a represalias en contra de él o incluso de su propia familia ; Por ultimo en la zona aguacatera, que lidera el municipio de Uruapan.

\subsection{BASES TEÓRICAS}

\subsubsection{Auge del aguacate}

México es el principal proveedor de todo el mercado, es decir, que tiene una gran aportación con el $45.95 \%$ del valor de todas las exportaciones mundiales.

\subsubsection{Actividades delictivas}

La gran industria del aguacate de México, en el estado de Michoacán, se convirtió en un objetivo principal para los cárteles de delincuencia. Más de 10 grupos delictivos quieren el control del comercio de aguacate alrededor queriéndose aprovechar de los dueños de los huertos. Todo esto se vio afectado por los asesinatos, secuestros, robo de producto etc., carteles de delincuencia, en el 2013 la situación se volvió tan insoportable para los productores aguacateros que tuvieron que armar ellos mismos sus grupos de autodefensa queriendo expulsar a los criminales y recuperar su producción.

Este nuevo enfoque del crimen organizado fue promovido por el famoso cártel de los Zetas y se extendió en respuesta a la declaración de guerra del gobierno de 2006 contra los narcotraficantes. Las 
fuerzas mexicanas, en apoyo de Estados Unidos, se dieron a la tarea de capturar o matar a los líderes de los cárteles. Pero esa estrategia fracasó cuando los grandes grupos criminales se fracturaron en organizaciones más pequeñas y ágiles que buscaban oportunidades delictivas donde pudieran encontrarlas. (Santa Cruz, D. 2016 Nueva Sociedad, “1185-1191”).

\subsubsection{Pérdidas económicas.}

De todas las problemáticas de los agricultores de aguacate una de la que más podemos destacar es la del robo del fruto por parte del crimen organizado, el robo de aguacate representa pérdidas económicas de más de 2 millones de pesos para los agricultores, por que registran de 7 a 10 camiones hurtados a la semana por parte del crimen organizado. Cada camión con una carga 8 toneladas de aguacate, cuyo costo promedio es de 200 mil peso

En las siguientes tablas se estiman las pérdidas económicas y robos del cultivo entre los años 2019 y 2020 . 
Figura 2

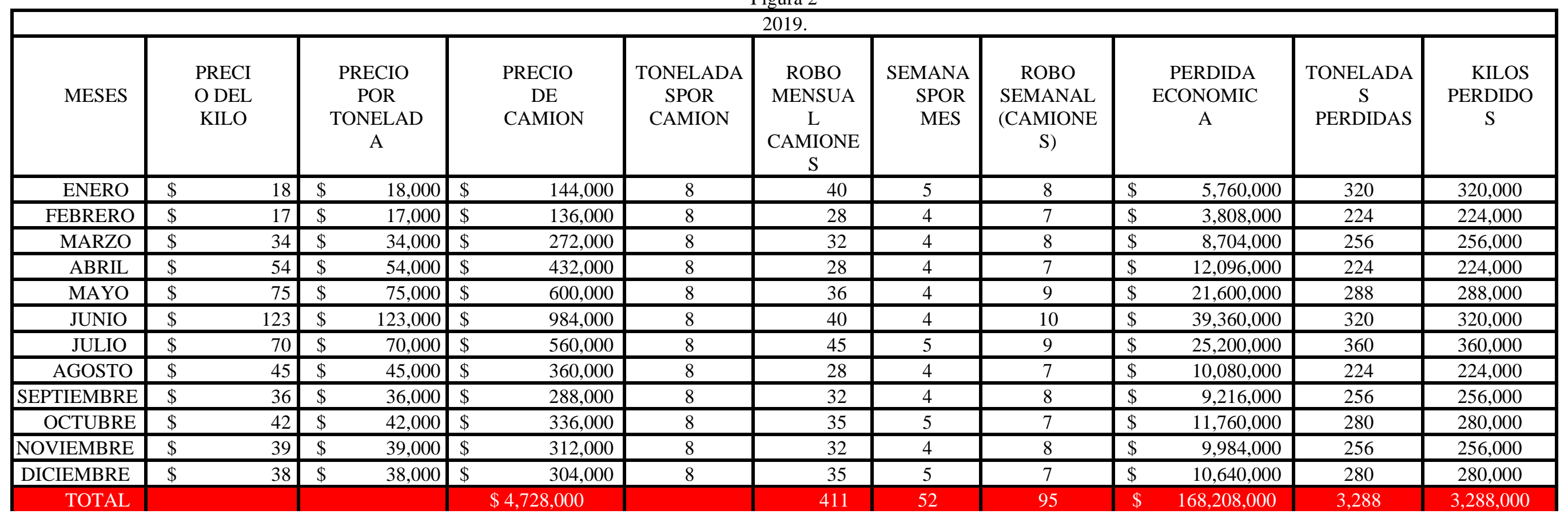


Figura 3

\begin{tabular}{|c|c|c|c|c|c|c|c|c|c|c|c|c|c|}
\hline \multicolumn{10}{|c|}{2020} & \multicolumn{4}{|c|}{ AUMENTO DE ROBO EN UN 30\% } \\
\hline MESES & & & & & $\begin{array}{l}\text { PRECIO DE } \\
\text { CAMION }\end{array}$ & $\begin{array}{c}\text { TONELADAS } \\
\text { POR } \\
\text { CAMION }\end{array}$ & $\begin{array}{c}\text { ROBO } \\
\text { MENSUAL } \\
\text { CAMIONES }\end{array}$ & $\begin{array}{c}\text { SEMANAS } \\
\text { POR } \\
\text { MES }\end{array}$ & $\begin{array}{c}\text { ROBO } \\
\text { SEMANAL } \\
(\text { CAMIONES) }\end{array}$ & & $\begin{array}{l}\text { RDIDA } \\
\text { NOMICA }\end{array}$ & $\begin{array}{c}\text { TONELADAS } \\
\text { PERDIDAS }\end{array}$ & $\begin{array}{c}\text { KILOS } \\
\text { PERDIDOS }\end{array}$ \\
\hline ENERO & $\$$ & 41 & $\$$ & 41,000 & $\$ 328,000$ & 8 & 52 & 5 & 10.4 & $\$$ & $17,056,000$ & 416.0 & 416,000 \\
\hline FEBRERO & $\$$ & 67 & $\$$ & 67,000 & $\$ 536,000$ & 8 & 36.4 & 4 & 9.1 & $\$$ & $19,510,400$ & 291.2 & 291,200 \\
\hline MARZO & $\$$ & 52 & $\$$ & 52,000 & $\$ \quad 416,000$ & 8 & 41.6 & 4 & 10.4 & $\$$ & $17,305,600$ & 332.8 & 332,800 \\
\hline ABRIL & $\$$ & 54 & $\$$ & 54,000 & $\$ \quad 432,000$ & 8 & 36.4 & 4 & 9.1 & $\$$ & $15,724,800$ & 291.2 & 291,200 \\
\hline MAYO & $\$$ & 59 & $\$$ & 59,000 & $\$ \quad 472,000$ & 8 & 46.8 & 4 & 11.7 & $\$$ & $22,089,600$ & 374.4 & 374,400 \\
\hline JUNIO & $\$$ & 42 & $\$$ & 42,000 & $\$ 336,000$ & 8 & 52 & 4 & 13 & $\$$ & $17,472,000$ & 416.0 & 416,000 \\
\hline JULIO & $\$$ & 48 & $\$$ & 48,000 & $\$ 384,000$ & 8 & 58.5 & 5 & 11.7 & $\$$ & $22,464,000$ & 468.0 & 468,000 \\
\hline AGOSTO & $\$$ & 54 & $\$$ & 54,000 & $\$ \quad 432,000$ & 8 & 36.4 & 4 & 9.1 & $\$$ & $15,724,800$ & 291.2 & 291,200 \\
\hline SEPTIEMBRE & $\$$ & 26 & $\$$ & 26,000 & $\$ \quad 208,000$ & 8 & 41.6 & 4 & 10.4 & $\$$ & $8,652,800$ & 332.8 & 332,800 \\
\hline OCTUBRE & $\$$ & 26 & $\$$ & 26,000 & $\$ \quad 208,000$ & 8 & 45.5 & 5 & 9.1 & $\$$ & $9,464,000$ & 364.0 & 364,000 \\
\hline NOVIEMBRE & $\$$ & 24 & $\$$ & 24,000 & $\$ \quad 192,000$ & 8 & 41.6 & 4 & 10.4 & $\$$ & $7,987,200$ & 332.8 & 332,800 \\
\hline DICIEMBRE & $\$$ & 24 & $\$$ & 24,000 & $\$ \quad 192,000$ & 8 & 45.5 & 5 & 9.1 & $\$$ & $8,736,000$ & 364.0 & 364,000 \\
\hline TOTAL & & & & & $\$ 4,136,000$ & & 534.3 & 52 & 123.5 & $\$$ & $182,187,200$ & $4,274.4$ & $4,274,400$ \\
\hline
\end{tabular}




\subsection{MARCO CONCEPTUAL}

\subsubsection{Generalidades}

La investigación se lleva a cabo debido a las pérdidas económicas que genera la delincuencia organizada a los agricultores de aguacate hass en Uruapan Michoacán, los robos de camiones de carga, parte de diferentes conceptos que se deben comprender dentro de la observación que se lleva a cabo. Se tomarán los conceptos más importantes del tema.

El auge de las exportaciones de aguacate se ha incrementado constantemente, es uno de los principales factores que conlleva a la delincuencia organizada al robo de dicho productos, ya que sus ganancias han sido crecientes en los últimos años, como resultado del consumo de este fruto.

\subsubsection{Pérdidas económicas}

Las pérdidas económicas son consecuencias de pérdidas o ingresos que afectan de manera negativa a la persona o empresa involucrada.

Aparte de robos y secuestro de unidades con conductores, también tenemos que sortear el exponer nuestra integridad física y la inseguridad imperante en las carreteras que debemos transitar para llegar al destino con la fruta ya empacada.

La jornada, Aguacateros en jaque por delincuencia organizada en Michoacán (2019).

\subsubsection{Agricultores}

Johnston y Kilby (1980), Weitz y Applebaum (1982) o el propio premio nobel T.W. Schultz (1967, 1969), Se refieren a la agricultura tradicional como a una estructura productiva propia de los primeros momentos del desarrollo económico. Se trata de un tipo de economía natural en la que "se utilizaba una energía renovable y unas materias primas que en su mayor parte se reproducían dentro del sector.

Ese concepto de economía natural encuadrado del de agricultura, procede de R. Luxemburg, para quien esa categoría aludía a dos características: la producción para consumo propio sobre la base de la no circulación de mercancías, y la sujeción de productores y medios de producción por la tradición y el derecho (R. Luxemburg 1978, 283).

Debido a los constantes cobros de derecho de piso que realiza la delincuencia organizada hacia los agricultores de aguacate, es como afecta de manera significativa en las pérdidas de ingresos, incluso de pérdidas de empleos. 


\subsubsection{Robo de camiones de aguacate}

"El impacto de la inseguridad en el transporte puede implicar enormes pérdidas para los dueños de la carga y los operadores, pero también puede poner en peligro a toda la comunidad" (Lucas Urciuoli, 2010).

Identifica la destrucción de la carga y la pérdida de mercancía como interrupciones del transporte. Particularmente, en el proceso de abastecimiento la "interrupción del transporte" es considerado por diversos autores (Young y Esqueda, 2005; Martner, Morales y de la Torre, 2005), como uno de los eslabones más vulnerables y de alto riesgo en la cadena logística (Cavinato. 2004).

México está en ese caso, pues la alta incidencia de robos en carretera reduce la competitividad logística del país, por las pérdidas económicas y los retrasos en el abastecimiento. Además, el aumento del nivel de violencia amenaza la integridad de los operadores; esto los motiva a dejar su trabajo por actividades de menor riesgo, agravando la actual carencia de operadores calificados de servicio federal de carga.

\subsubsection{Delincuencia organizada que afecta a los agricultores de aguacate}

Para la Ley Federal Contra la Delincuencia Organizada, en el artículo 2, Cuando tres o más personas se organicen de hecho para realizar, en forma permanente o reiterada, conductas que por sí o unidas a otras, tienen como fin o resultado cometer alguno o algunos de los delitos siguientes, serán sancionadas por ese solo hecho, como miembros de la delincuencia organizada.

Entre los productores de aguacate Michoacano es un secreto a voces la presencia del crimen organizado. La multimillonaria industria del aguacate se ha convertido en el objetivo principal para grupos delictivos. Los cárteles se han encargado de incautar granjas, cobrar "rentas" e incluso limpiar bosques protegidos para plantar sus propios huertos de aguacate (Gabriel Villaseñor, presidente de la APEAM, 2020).

\subsubsection{Aguacate Hass}

Es una fruta con un peso de 150 a 350 gramos habitualmente, tanto el fruto como la semilla son relativamente pequeños a comparación del aguacate fuerte, cuando la fruta está madura la piel es de color púrpura oscuro casi negro y cede ante una presión suave, por lo que el consumidor reconoce el momento óptimo para consumirla, cuando está listo para servir se convierte en blanco y verde en la parte media del fruto interno. En general, el árbol de esta variedad florece una sola vez al año, desde inicios a mediados de primavera y, dependiendo de la variedad y la acumulación térmica del lugar, puede demorar entre 6 y 11 meses en madurar. La variedad Hass, especialmente en climas frescos, puede mantenerse en el árbol 
una vez madura, durante varios meses sin mayor deterioro aparente, lo cual permite extender enormemente el período de cosecha (CDMB. 2013).

\subsubsection{Auge por la comercialización del aguacate Hass.}

El aguacate hass se encuentra ubicado como uno de los frutos más comercializados a nivel internacional. De acuerdo con el crecimiento que aporte al país en las exportaciones y las ganancias que benefician a México, es como el aguacate se denomina "Oro Verde" porque es uno de los productos que cotiza en la bolsa mexicana de valores.

Considerada la estrella del campo mexicano, el aguacate es uno de los frutos preferidos de la cocina internacional. Países como Japón, Canadá, China y la Unión Europea son grandes consumidores de aguacate (Portal Frutícola, 2013).

\subsubsection{Auge}

Se define al auge económico como aquella fase del ciclo económico en el cual las variables principales alcanzan el grado máximo de expansión porque se ha generado un mayor empleo de los factores de producción y también porque han mejorado las condiciones de los mercados (Florencia Ucha, 2010).

\subsubsection{Comercialización}

Dar a un producto condiciones y organización comercial para su venta (LAROUSSE, 2008).

Entre los diferentes tipos de aguacate y de acuerdo con las principales propiedades con las que cuenta el aguacate hass es uno de los tipos de aguacate en altos índices de comercialización y exportación, a nivel nacional e internacional, durante el año y con más auge, esto generando un desarrollo económico.

\section{TIPO DE DISEÑO DE INVESTIGACIÓN.}

La investigación presente es un tipo de investigación experimental cuantitativa, ya que las variables son cuantificables y así se estudiarán las pérdidas económicas de lo que lleva el año 2021. Se tomaran datos de años anteriores para realizar un estudio de series de tiempo.

Se analizara la causa y el efecto de las variables, con la recopilación de datos sobre las pérdidas económicas ante los robos del aguacate Hass. Serán manipulables las variables de la investigación para cumplir con los objetivos establecidos al inicio de la investigación.

Para el nivel de estudio que se llevará acabo, será la predicción de las variables dependientes. 


\subsection{NIVEL DE ESTUDIO}

Para predecir las pérdidas económicas que género la delincuencia organizada a los agricultores de aguacate Hass del municipio de Uruapan Michoacán para el año 2021. Se Utilizara la técnica de series de tiempo como herramienta en la predicción de pérdidas económicas.

El estudio de series de tiempo tiene como objetivo central, desarrollar modelos estadísticos que expliquen el comportamiento de una variable aleatoria que varía con el tiempo, y que permiten estimar pronósticos futuros de dicha variable aleatoria.

La importancia de construir modelos predictivos, muestra de una variable endógena, que se entiende como variable a predecir, de esta forma se requiere proyectar las pérdidas económicas que género la delincuencia organizada a los agricultores de aguacate hass, para el año 2021. Debido a los robos de camiones de aguacate que se presentan continuamente se estimará y se graficarán las pérdidas económicas, tomando como base datos de años anteriores (2019 y 2020). Se realizarán los cálculos para llegar a una estimación y a su vez predecirlas.

\subsection{ENFOQUE DE LA INVESTIGACIÓN}

Dentro de la investigación se empleará un enfoque cuantitativo, puesto que el objetivo es realizar una estimación de las pérdidas económicas, debido al robo de aguacate en el municipio de Uruapan para el año 2021, tomando como referencia los años anteriores; este enfoque se encarga de la recolección y análisis de datos para responder a la pregunta de investigación y probar la hipótesis ya establecida, se utilizan operaciones numéricas, mediciones y se hace uso de la estadística para establecer con exactitud patrones para predecir el comportamiento de las variables.

\subsection{MÉTODO DE LA INVESTIGACIÓN}

El método que se empleara para la predicción de las pérdidas económicas, el cual será pronóstico de series de tiempo.

El análisis parte del año 2019, en donde afirma el artículo publicado el 18 de junio de 2019 en la jornada.

La vocalía de la Asociación de Productores y Empacadores de Aguacate afirmó que en los últimos dos años en promedio se robaban entre siete y 10 camiones de aguacate de exportación a la semana, cada uno de ellos con ocho toneladas del fruto, con costo de 200 mil pesos por unidad.

En base a ello y con datos corroborados de la APEAM, donde se obtuvieron los precios del aguacate en los meses correspondientes de cada año, realizando así, tablas de pérdidas de los años anteriores. (Páginas 5 y 6). 
Para los resultados del año 2021, se tomaran el primer trimestre y así obtener el promedio y realizar el análisis de tendencia con series de tiempo.

obtener los intervalos de frecuencia de las pérdidas económicas

\subsection{TÉCNICA DE INVESTIGACIÓN}

La técnica empleada, como ya antes se había mencionado son las series de tiempo, se analizarán y se emplearan para la obtención de las pérdidas económicas más exactas utilizando cada uno de los métodos de series de tiempo.

Métodos de series de tiempo.

- Análisis de tendencia lineal.

- Análisis de tendencia cuadrática.

- Análisis de tendencia exponencial.

- Análisis de tendencia curva S.

- Descomposición de series de tiempo (Multiplicativo * tendencia más estacional).

- Descomposición de series de tiempo (Aditivo * tendencia más estacional).

- Descomposición de series de tiempo (Multiplicativo * estacional solamente).

- Descomposición de series de tiempo (Aditivo * estacional solamente).

- Suavización exponencial simple.

- Suavización exponencial doble.

- Método de Winter.

Para el procedimiento de cada uno de los métodos de series de tiempo, se utiliza un software llamado minitab, es un software estadístico que ayuda a las predicciones futuras, en el caso de las pérdidas de aguacate Hass, se inserta todos los datos de los años 2019 y 2020 con la diferencia que se cambia los meses por números, ya que el software no reconoce los meses como datos, lo cual significa que el mes de enero del año 2019 será el número 1 y febrero del mismo años será el número 2 y así consecutivamente.

Ya teniendo las series de tiempo previamente pronosticadas, se procede a comprar los MAPES de cada uno de los métodos de series de tiempo que dieron como resultado, para la selección del método con el menor margen de error para el análisis.

Se recuerda que el MAPE es el error porcentual absoluto medio, significa que el margen de error que se obtiene al pronosticar un dato real.

El MAD o la desviación absoluta, es el promedio de las desviaciones en unidades. Y por último el MSD o desviación cuadrática media es la varianza del error pronosticado en promedio. 
Figura 17 Relación del MAPE, MAD Y MSD de las pérdidas económicas

\begin{tabular}{|c|c|c|c|}
\hline Método. & МАРE. & MAD. & MSD. \\
\hline \multicolumn{4}{|l|}{ Análisis de tendencia lineal. } \\
\hline & 5.24 & 5.9 & 5.41 \\
\hline \multicolumn{4}{|l|}{ Análisis de tendencia cuadrática. } \\
\hline & 4.22 & 5.09 & 8.85 \\
\hline \multicolumn{4}{|l|}{ Análisis de tendencia exponencial. } \\
\hline & 4.36 & 5.64 & 5.77 \\
\hline \multicolumn{4}{|l|}{ Análisis de tendencia curva $\mathrm{S}$. } \\
\hline & ERROR & ERROR & ERROR \\
\hline \multicolumn{4}{|l|}{$\begin{array}{l}\text { Descomposición de series de tiempo (Multiplicativo *tendencia más } \\
\text { estacional). }\end{array}$} \\
\hline & 3.79 & 3.63 & 3.83 \\
\hline \multicolumn{4}{|l|}{ Descomposición de series de tiempo (Aditivo * tendenciamás estacional). } \\
\hline & 3.9 & 3.72 & 3.91 \\
\hline Descomposición de series de tiempo (Multiplicativo *estacional solamente). & 3.85 & 3.64 & 3.84 \\
\hline Descomposición de series de tiempo (Aditivo * estacionalsolamente). & & & \\
\hline & 4.06 & 3.8 & 3.93 \\
\hline Suavización exponencial simple. & & & \\
\hline & 3.12 & 4.72 & 4.25 \\
\hline Suavización exponencial doble. & & & \\
\hline & 3.59 & 5.01 & 4.6 \\
\hline Método de Winter. & & & \\
\hline & 3.27 & 4.89 & 5.92 \\
\hline
\end{tabular}

En la tabla anterior, los datos señalados con rojo serán el método que menos se adapte al objetivo de predicción de las pérdidas económicas, que se obtiene un margen de error muy alto. Los métodos de color amarillo y naranja, cuentan con un margen de error promedio y pueden ser los posibles métodos seleccionados, es decir, que no es un margen de error alto ni bajo, sin en cambio los métodos seleccionados que se muestran de color verde, son los de menor rango de error y

\subsection{ANÁLISIS DEL MÉTODO SUAVIZACIÓN EXPONENCIAL SIMPLE PARA LAS PÉRDIDAS ECONÓMICAS}

\section{Método - Datos tomados con anterioridad}

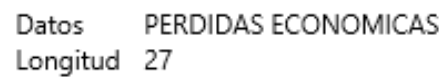

Datos PERDIDAS ECONOMICAS

Longitud 27

\section{Medidas de exactitud}

$\begin{array}{ll}\text { MAPE } & 3.12200 \\ \text { MAD } & 4.72303 \\ \text { MSD } & 4.25928\end{array}$

\section{Pronósticos}

\begin{tabular}{|c|c|}
\hline Período & Pronóstice Inferior Superior \\
\hline Abril & $20,232.6398,661.41731,803.861$ \\
\hline Mayo & $20,232.6398,661.41731,803.861$ \\
\hline Junio & $20,232.6398,661.41731,80$ \\
\hline Julio & 41731,8 \\
\hline Agosto & 1731,8 \\
\hline Septiembre & $20,232.6398,661.41731,803.861$ \\
\hline Octubre & $20,232.6398,661.417 \quad 31,803.861$ \\
\hline & \\
\hline Diciembre & $98,661.41731 .803 .861$ \\
\hline
\end{tabular}

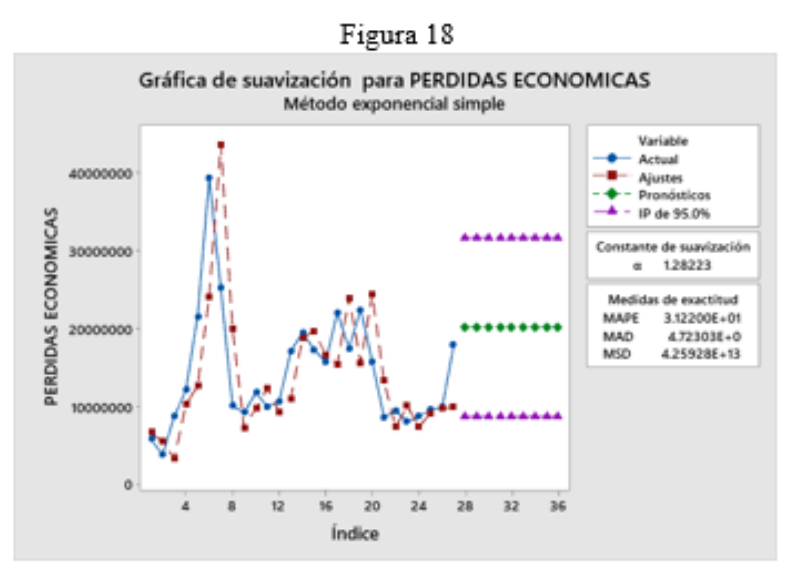


El método indica que la pérdida económica, pronostica que para el mes de abril del 2021 se tendrá una pérdida económica de \$20, 232,639.00 pesos mexicanos, sin embargo las pérdidas no superaran los $\$ 31,803,861.00$ pesos mexicanos. El pronóstico muestra un mínimo de pérdida por la cantidad de $\$ 8$, 661,417.00 pesos mexicanos.

Como se expresa en la gráfica (figura 18), que la línea de tendencia es recta y predice para los peses posteriores.

\subsection{ANÁLISIS DEL MÉTODO DE WINTER PARA LAS PÉRDIDAS ECONÓMICAS}

Este método, tomando en cuenta la comparación de MAPE de la figura 17, es la más certera para tener un error minino de pronóstico, es más conciso en las variaciones de pérdidas económicas de mes a mes para el año 2021.

\section{Método}

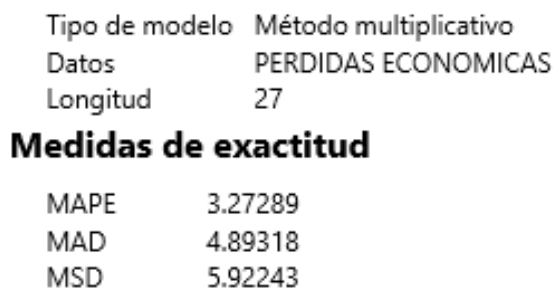

\section{Pronósticos}

\begin{tabular}{lrrrrr} 
Período & & Pronóstico & Inferior & & Superior \\
\cline { 1 - 1 } Abril & & $12,691.826$ & 703.756 & $24,679.897$ \\
Mayo & & $19,558.063$ & $7,382.179$ & $31,733.947$ \\
Junio & & $24,757.416$ & $12,372.118$ & $37,142.713$ \\
Julio & & $20,138.079$ & $7,522.845$ & $32,753.314$ \\
Agosto & & $10,674.645$ & $-2,189.951$ & $23,539.240$ \\
Septiembre & & $7,236.594$ & $-5,895.679$ & $20,368.867$ \\
Octubre & & $8,403.538$ & $-5,013.633$ & $21,820.709$ \\
Noviembre & & $6,973.476$ & $-6,744.741$ & $20,691.692$ \\
Diciembre & & $7,379.072$ & $-6,655.299$ & $21,413.444$
\end{tabular}

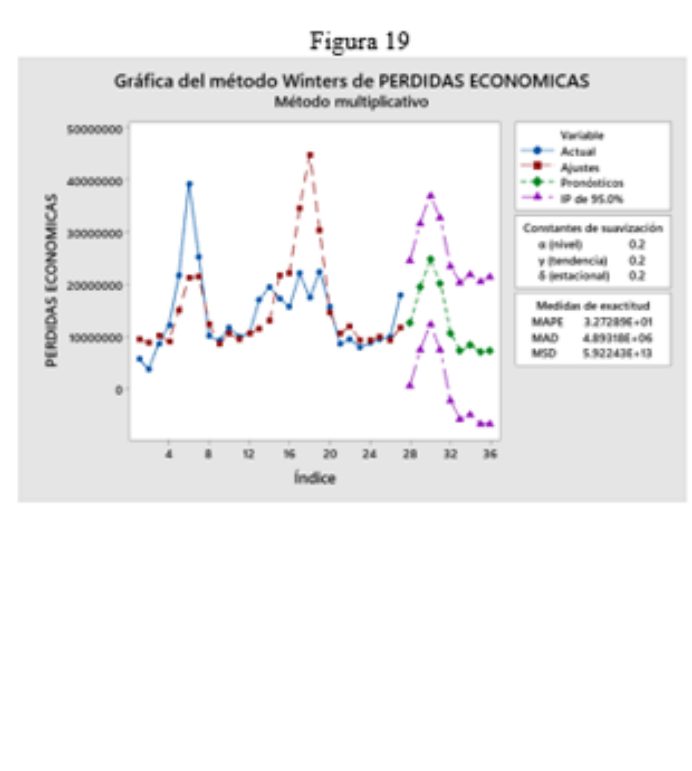

Utilizando el método Winter indica que en el mes de abril pronostica una pérdida de $\$ 12$, $691,926.00$ pesos mexicanos, con un rango inferior de $\$ 703,756.00$, es decir, que es la cantidad mínima de pérdida económica que se pronostica en ese mes y no mayor a los

$\$ 24,679,897.00$ de pesos mexicanos. Y de la misma manera con los meses próximos, el pronóstico ya calculado, es la estimación que se pronostica para los meses próximos. En pocas palabras el límite inferior es lo mínimo de pérdida que se pronostica en el mes y el límite superior es el rango más alto que puede llegar en pérdidas económicas.

En los límites inferiores se muestran números negativos en los meses de agosto, septiembre, octubre, noviembre y diciembre quiere decir que en esos meses si se pronostican perdidas pero también se pronostican que no puede ver perdidas. 
Se expresa de una manera gráfica (figura 19) y tener un mejor entendimiento de la línea de tendencia no constante como en la gráfica anterior (figura 18).

\subsection{UNIVERSO Y MUESTRA}

Universo: Estado de Michoacán Uruapan.

Muestra: Agricultores de aguacate Hass, del municipio de Uruapan Michoacán.

La manera de elección del universo y la muestra, para la investigación se basa en la pregunta de investigación, ya que hace referencia al Estado de Michoacán y el municipio de Uruapan, por lo tanto el universo es el Estado de Michoacán y la muestra, son los agricultores de aguacate Hass en el municipio de Uruapan Michoacán, la forma de recolección de datos para la realización del pronóstico de las pérdidas económicas, fue mediante la consulta de los reportes dados por APEAM (Asociación de productores y empacadores, exportadores de aguacate de México).

La muestra seleccionada es el municipio de Uruapan, ya que es el principal productor de aguacate Hass dentro del Estado de Michoacán y de igual manera los principales afectados son los agricultores, por la delincuencia organizada y el robo de toneladas del fruto, ya que es la variable fundamental de la investigación.

\section{APLICACIONES DE INSTRUMENTOS DE MEDICIÓN.}

Los métodos de series de tiempo ya analizados anteriormente dentro de la metodología, para el análisis de resultados el método de suavización exponencial, no es una opción para la comprobación de la hipótesis ya que supera el rango de las pérdidas económicas mencionadas.

Para el método de Winter, es el método que comprueba la hipótesis ya que muestra el análisis de las pérdidas económicas más exactas.

\subsection{ANÁLISIS DE RESULTADOS}

Para la obtención de resultados se eligieron los métodos más factibles para la comprobación de la hipótesis el cual es el método de suavización exponencial y Winter, ambos métodos predicen resultados, en este tema las pérdidas económicas, en la metodología se analizó el crecimiento de las pérdidas económicas desde el año 2019 al 2021.

\subsection{MÉTODO DE SUAVIZACIÓN EXPONENCIAL SIMPLE}

Al tomar los resultados dados por el calculo de las predicciones utilizando este método, se realizan los cálculos para obtener la pérdida 
económica total de todo el año 2021. Como se muestra en la siguiente tabla.

La tabla muestra un total de $\$ 219,533,751.00$ millones de pesos como pérdida que se obtendrá para el año 2021, por lo tanto, utilizando este método, no comprueba la hipótesis, ya que sobre pasa por $\$ 19,533,751.00$ millones.

Para tener más claro el comportamiento de este método, se ve en la siguiente grafica.

\begin{tabular}{|c|c|c|c|}
\hline \multicolumn{4}{|c|}{ EXPONENCIAL SIMPLE } \\
\hline \multicolumn{4}{|c|}{2021} \\
\hline MESES & \multicolumn{2}{|c|}{$\begin{array}{c}\text { PERDIDA } \\
\text { ECONOMICA }\end{array}$} & TRIMESTRES \\
\hline ENERO & $\$$ & $9,568,000$ & \\
\hline FEBRERO & $\$$ & $9,900,800$ & \\
\hline MARZO & $\$$ & $17,971,200$ & \\
\hline \multicolumn{3}{|r|}{ Subtotal } & $37,440,000$ \\
\hline ABRIL & $\$$ & $20,232,639$ & \\
\hline MAYO & $\$$ & $20,232,639$ & \\
\hline JUNIO & $\$$ & $20,232,639$ & \\
\hline \multicolumn{3}{|r|}{ Subtotal } & $60,697,917$ \\
\hline JULIO & $\$$ & $20,232,639$ & \\
\hline AGOSTO & $\$$ & $20,232,639$ & \\
\hline SEPTIEMBRE & $\$$ & $20,232,639$ & \\
\hline \multicolumn{3}{|r|}{ Subtotal } & $60,697,917$ \\
\hline OCTUBRE & $\$$ & $20,232,639$ & \\
\hline NOVIEMBRE & $\$$ & $20,232,639$ & \\
\hline DICIEMBRE & $\$$ & $20,232,639$ & \\
\hline \multicolumn{3}{|r|}{ Subtotal } & $60,697,917$ \\
\hline & & TOTAL & $219,533,751$ \\
\hline
\end{tabular}

En la grafica se muestra que la línea de tendencia va en aumento, para el primer trimestre del año 2021, son datos obtenidos por la APEAM, lo cual permite realizar las predicciones. Los otros tres trimestres están a la par ya que los resultados son los mismos.

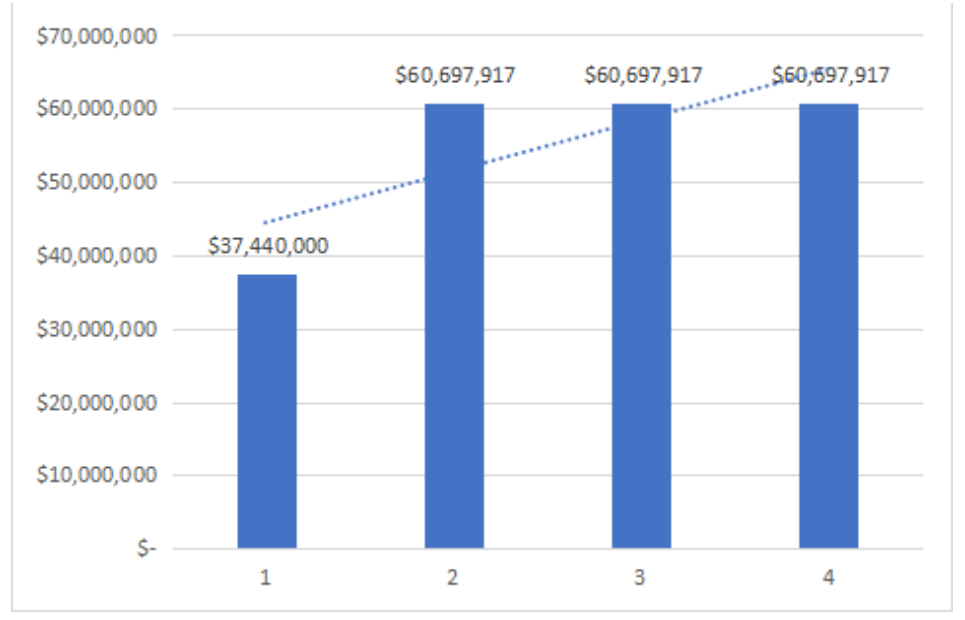

\subsection{MÉTODO WINTER}

Para el método Winter, da resultados totalmente diferentes al método anterior ya que son resultados totalmente diferentes, por lo cual se realizan los cálculos para la obtención de la pérdida económica.

Con este método es totalmente diferente al anterior, ya que los resultados por trimestres son muy diferentes, ya que no se obtienen resultados iguales. 
En la tabla de resultados se muestra que en el segundo trimestre es donde más se obtuvieron pérdidas económicas.

Como total de las pérdidas económicas en el año 2021, se pronostican $\$ 155,252,709.00$, por lo tanto, con el método Winter se demuestra la hipótesis de la investigación, ya que se encuentra dentro del rango.

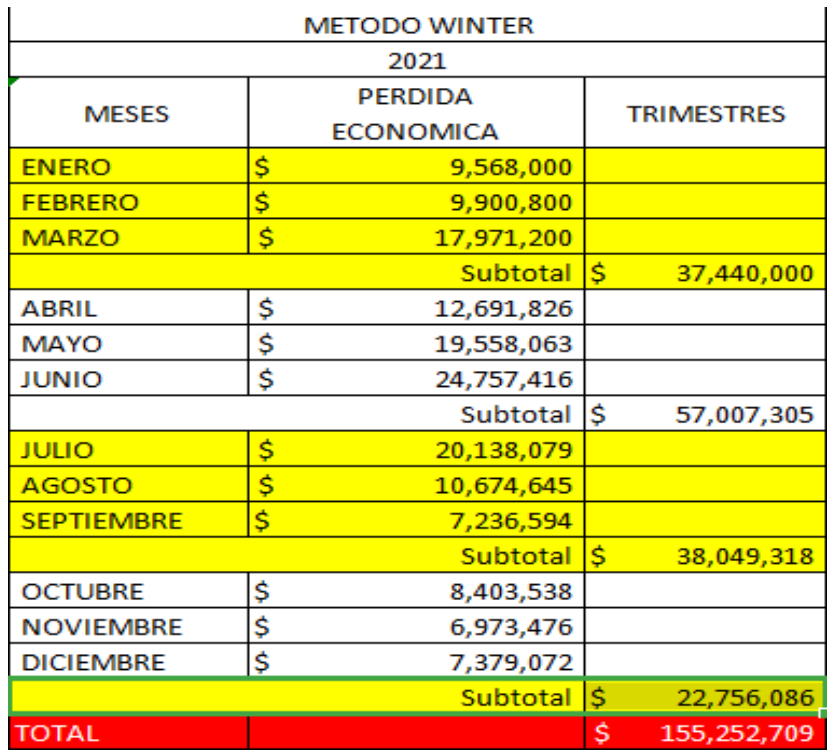

En la gráfica se puede observar que la línea de tendencia al final del año va en declive. De igual manera el primer trimestre del año 2021, son datos obtenidos por la APEAM.

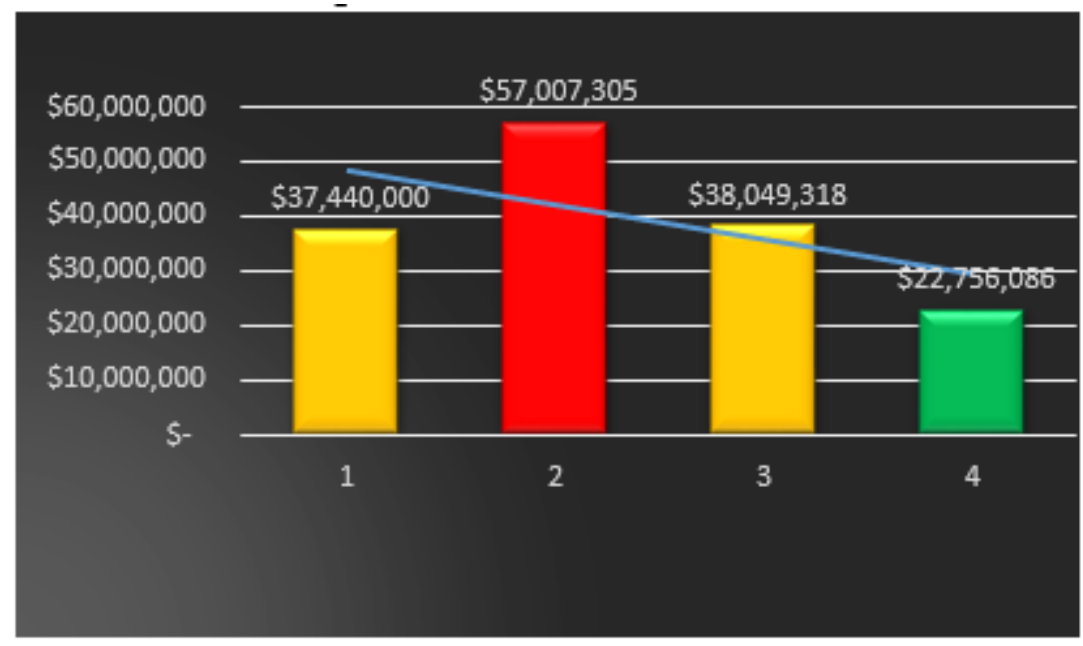

\section{CONCLUSIONES}

En este trabajo se Proyectaron las pérdidas económicas, que generará la delincuencia organizada a los agricultores de aguacate Hass del municipio de Uruapan Michoacán para el año 2021.

Lo más importante de haber realizado una proyección sobre las pérdidas económicas, debido a que se emplearon diversos métodos de series de tiempo. Buscando así el más adecuado, demostrando las 
pérdidas económicas sobre el robo de aguacates para el año 2021 (paginas 14:24) Y relacionando así los diferentes resultados de cada uno de ellos, seleccionando de esta manera el que demuestre un menor margen de error.

Asimismo, lo que más ayudo a realizar esta proyección fue el acceso a el historial de precios de aguacate por medio de la página oficial APEAM (Asociación de Productores y Empacadores Exportadores de Aguacate de México) y el uso de un software avanzado en estadística.

Gracias ello se pudieron hacer los cálculos y conversiones correspondientes.

Lo complejo al momento de la realización de la estimación fue la interpretación de datos, esto porque cada método tiene un resultado distinto, debido a que las series de tiempo se usan para distintos fines, sin embargo, en este trabajo se requería el menor error absoluto medio porcentual, siendo la menor medida de precisión para la predicción de esta investigación en términos estadísticos de igual manera en la recolección de información.

En el desarrollo de esta investigación se recopilaron datos cuantitativos de acuerdo con los antecedentes de años pasados los cuales muestran un auge en el incremento de robo de aguacates teniendo como referencia las toneladas y los robos a los camiones semanalmente en cada mes de año en base a los precios.

Por su parte en cuanto a la recolección de información sobre los camiones fue una parte muy difícil, ya que no se contaba con datos sobre el total de camiones exportadores en el mes, trimestres o año para tener una población total, sin embargo, las conversiones correspondientes se basaron en nuestros antecedentes y noticias investigadas.

Esta investigación fue controversial y difícil, ya que existe poca información cuantitativa sobre el robo del robo de camiones de aguacate en el municipio de Uruapan Michoacán, Teniendo así no una referencia exacta sobre la cantidad de dinero perdido respecto al tema, basándonos en nuestros conocimientos adquiridos como gestores empresariales, se pudo realizar dicho pronóstico, teniendo así, un dato relevante y muy alarmante respecto a dichas perdidas.

Para finalizar, empleamos los conocimientos adquiridos en este informe, lo cual proporciono diferentes maneras de dar solución con propuestas como la seguridad y apoyo que se puede otorgar de parte de gobierno a los agricultores de Uruapan Michoacán, ya que se sabe que estas personas no tienen mucho respaldo con respecto al gobierno y tienen que sufrir tanto la inseguridad como la merma de su cosecha, llegando al grado de homicidio. Con esta investigación se espera que se pueda brindar apoyo y priorizar el tema respecto al robo de camiones aguacateros, ya que es una fuerte suma de dinero con respecto a un solo producto agrícola, esto nos da una leve idea de que tantas pérdidas millonarias tiene nuestro país con respecto a otros productos que México exporta. 


\section{REFERENCIAS}

Aguilar Armando, (2020), Los grandes retos del aguacate mexicano, México. Obtenido de: https://goula.lat/los-grandes-retos-del-aguacate-mexicano/. El 26 de febrero del 2021.

Agencia Reforma, (2020), Productores de aguacate aseguran que delincuentes les roban millones en mercancía, México. Obtenido de:

https://www.elimparcial.com/economianegocios/Productores-de-aguacate- aseguran-que-delincuentesles-roban-millones-en-mercancia-20190920-0014.html. El 25 de febrero del 2021.

APEAM, (2021), Aguacate mexicano, México. Obtenido de: http://www.apeamac.com/. El 26 de febrero del 2021.

Béjar, Ayala, Rico, Oropeza, Isidro, Padilla, Soto, Ayala y Rangel, (2018), El aguacate; el gran oro verde. Obtenido de: https://www.univa.mx/zamora/wp- content/uploads/sites/3/2019/07/articulo-elaguacate.pdf. El 28 de febrero del 2021.

CDMB. (2013). La CDMB implementa Plan Ambiental en cultivo de aguacate Hass, en Tona.

Obtenido de CDMB:

http://www.cdmb.gov.co/web/ciudadano/prensa/noticias/item/1849-la-cdmb- implementaplanambiental-en-cultivo-de-aguacate-hass-en-ton. El 03 de marzo del 2021.

Ciudad Guzmán y Uruapan, México. Revista de Ciencias Sociales (Ve), XXVI ((Número especial 2), 414425

Colarte Cortés “Arrebata a crimen el manejo de oro verde “en El Universal 17/01/2016

DEMOS, Desarrollo de Medios, SA de CV (2019, 18 de junio). La Jornada: Robo de aguacate, también entre los productores: SSP. La Jornada. https://www.jornada.com.mx/2019/06/18/economia/020n1eco. El 03 de marzo del 2021.

Estrada Arturo, (2018), Van 50 mil toneladas de aguacate perdidas por conflicto entre productores, México. Obtenido de: https://www.elfinanciero.com.mx/nacional/van-50-miltoneladas-de-aguacate- perdidas-por-conflicto-entre-productores. El día 26 de febrero del 2021.

La Ley Federal Contra la Delincuencia Organizada, (2009) "La delincuencia organizada". Obtenido: https://mexico.justia.com/federales/leyes/ley-federal-contra- la-delincuencia-organizada/tituloprimero/capitulo-unico/\#articulo-1.

Linthicum Kate, (2019), La guerra del narco para controlar la multimillonaria industria del aguacate en México, Estados Unidos. Obtenido de:

https://www.latimes.com/espanol/mexico/articulo/2019-11-21/mexico-cartel- violencia-aguacates. El 25 de febrero del 2021.

La jornada, (2019), “Aguacateros en jaque por delincuencia organizada en Michoacán”. Obtenido de: https://www.jornada.com.mx/2019/06/16/economia/017n1eco

Larousse Editorial S.L, (2008) $1^{\text {a }}$ Edición 1920, Castellano Libro. Obtenido de: https://editorial.tirant.com/es/libro/el-pequeno-larousse-2008-edicion-especial- 9788480167819. 
Luxemburg, R. (1978). La acumulación del capital. México DF: Grijalbo. P 283 Martínez Alonso, (2020), El aguacate es la nueva "droga" que quiere controlar el crimen organizado en México.

Obtenido de: https://www.gq.com.mx/entretenimiento/articulo/el-aguacate-es-la-nuevadroga- del-crimen-organizado-en-mexico. El 26 de febrero del 2021.

Naredo, J.M. (1986): "La agricultura española en el desarrollo económico", en GARRABOU, R.; BARCIELA; C. JIMÉNEZ, J.1. (Eds).Historia agraria de la España contemporánea (IlI). Crítica, Barcelona, p.458.

Portal Frutícola., (2013) "México mantiene estimación de exportación de aguacate a EE. UU” [En línea]. México, Obtenido de: http://www.portalfruticola.com/noticias/2013/11/08/mexico-mantieneestimacionde- exportacion-de-aguacate-a-ee-uu/?pais=otrospaises

S. Cruz David" Franquicias para el delito" (2016) Nueva Sociedad N²63 Vanguardia, (2021), Ni el Super Bowl salva las exportaciones de aguacate mexicano, México. Obtenido de: https://vanguardia.com.mx/articulo/ni-el-super- bowl-salva-las-exportaciones-de-aguacate-mexicano. El 26 de febrero del 2021.

Villaseñor, Gabriel (2010) presidente de la Asociación de Productores y Empacadores Exportadores de Aguacate de México, "delincuencia organizada que afecta a los agricultores de aguacates". Obtenido de: http://www.apeamac.com/tag/gabriel-villasenor/. El 03 de marzo del 2021.

Valdez Zepeda Andrés, (2001), “Oro verde”, Educación e inseguridad en ciudades medias, México. Obtenido de: https://dialnet.unirioja.es/servlet/articulo?codigo=7599954. El 03 de marzo del 2021. Zea Francisco, (2019), ¿Por qué el aguacate mexicano es considerado "oro verde"?, México, https://www.youtube.com/watch?v=bc2E6vPrhNU. El 20 de febrero del 2021. 


\section{NOTA DE RODAPÉ}

Valdez, A. y Sánchez, M. (2020). “Oro verde”, educación e inseguridad en ciudades medias:

Ciudad Guzmán y Uruapan, México. Revista de Ciencias Sociales (Ve), XXVI((Número especial 2),414425

C.Olarte Cortés “Arrebata a crimen el manejo de oro verde "en El Universal 17/01/2016

S. Cruz David” Franquicias para el delito" (2016)Nueva Sociedad N²63

4

Grupos de Autodefensa: Los Grupos de Autodefensa o Guardias Comunitarias son un grupo de civiles armados, mayoritariamente aguacateros o limoneros que toman las armas para defenderse de los ataques de los cárteles mexicanos

\section{OEM -INFOMEX}

DEMOS, Desarrollo de Medios, SA de CV (2019, 18 de junio). La Jornada: Robo de aguacate, también entre los productores: SSP. La Jornada. https://www.jornada.com.mx/2019/06/18/economia/020n1eco

La jornada, (2019), “Aguacateros en jaque por delincuencia organizada en Michoacán”.Obtenido de : https://www.jornada.com.mx/2019/06/16/economia/017n1eco Naredo, J.M. (1986): "La agricultura española en el desarrollo económico", en GARRABOU, R.; BARCIELA; C. JIMÉNEZ, J.1. (Eds).Historia agraría de la España contemporánea (IlI). Crítica, Barcelona, p.458.

Luxemburg, R. (1978). La acumulación del capital. México DF: Grijalbo. P 283

La Ley Federal Contra la Delincuencia Organizada, (2009) "La delincuencia organizada". Obtenido: https://mexico.justia.com/federales/leyes/ley-federal-contra-ladelincuencia-organizada/tituloprimero/capitulo-unico/\#articulo-1.

Villaseñor, Gabriel (2010) presidente de la Asociación de Productores y Empacadores Exportadores de Aguacate de México, "delincuencia organizada que afecta a los agricultores de aguacates". Obtenido de: http://www.apeamac.com/tag/gabriel-villasenor/.

CDMB. (2013). La CDMBimplementa Plan Ambiental en cultivode aguacate Hass, en Tona. Obtenido de CDMB:

http://www.cdmb.gov.co/web/ciudadano/prensa/noticias/item/1849-la-cdmb-implementaplan-ambientalen-cultivo-de-aguacate-hass-en-ton.

Larousse Editorial S.L, (2008) $1^{\text {a }}$ Edición 1920, Castellano Libro. Obtenido de: https://editorial.tirant.com/es/libro/el-pequeno-larousse-2008-edicion-especial- 9788480167819.

Portal Frutícola., (2013) "México mantiene estimación de exportación de aguacate a EE.UU” [En línea]. México, Obtenido de: http://www.portalfruticola.com/noticias/2013/11/08/mexico-mantieneestimacionde-exportacion-de-aguacate-a-ee-uu/?pais=otrospaises 